\title{
Manuel d'hémodynamique appliquée en anesthésie réanimation et médecine péri-opératoire
}

\author{
Collège National des Enseignants d'Anesthésie et de Réanimation; Marc-Olivier Fischer, \\ Yazine Mahjoub (coordonnateurs). Collection CM, Presses Universitaires François- \\ Rabelais, 2020, 432 pages. ISBN 978-2-86906-755-4
}

\author{
André St-Pierre, MD, FRCPC
}

Received: 1 February 2021 / Accepted: 2 February 2021 / Published online: 2 March 2021

(C) Canadian Anesthesiologists' Society 2021

Le transport des éléments nécessaires au métabolisme entre les différents organes constitutifs est une des fonctions essentielles de l'organisme pour assurer l'homéostasie entre le milieu interne et son environnement. La connaissance de la physiologie de la circulation, des limites de son adaptation, des modifications pathologiques et de leurs traitements est un domaine en constant développement. La diffusion de ces concepts, permettant leur application au cours de la formation médicale et la mise à jour de toutes ces connaissances sont des jalons essentiels à la prise en charge des patients en situation clinique d'instabilité physiologique. C'est pour permettre aux cliniciens d'avoir un ouvrage qui renferme les notions essentielles sur ce sujet que le Collège national des enseignants d'anesthésie et de réanimation de France a publié le Manuel d'Hémodynamique appliquée en Anesthésie Réanimation et Médecine péri-opératoire.

Ce manuel est l'œuvre de 38 auteurs provenant de 15 centres hospitaliers universitaires français. Les professeurs Fischer et Mahjoub ont utilisé cet outil pédagogique pour regrouper en cinq thèmes les connaissances hémodynamiques qui sont développées par les différents auteurs des 32 chapitres du manuel: Pathophysiologie appliquée, Outils diagnostiques, Outils thérapeutiques, Situations cliniques et Médecine péri-opératoire.

Chaque chapitre débute par l'identification de pointsclés pertinents qui seront par la suite développés dans les différentes sections caractérisées par leur concision. Les tableaux et les figures élaborés par les auteurs sont clairs et

\footnotetext{
A. St-Pierre, MD, FRCPC ( $\varangle)$

Département d'anesthésiologie et de soins intensifs, Université Laval, Québec, QC, Canada

e-mail: andre.st-pierre@fmed.ulaval.ca
}

leur juxtaposition au texte ajoute à l'explication et à la synthèse des propos discutés. La monochromie de leur édition permet de capter avec facilité les sujets en discussion. Quelques reproductions d'imagerie auraient cependant bénéficié d'être plus contrastées afin de faciliter la démonstration recherchée.

Les chapitres sont courts, de 7 à 20 pages. Ils reflètent bien l'objectif des coordonnateurs de ce manuel de fournir aux étudiants des spécialités d'Anesthésie Réanimation et de Médecine péri-opératoire une approche structurée des différents aspects théoriques et pratiques du travail clinique auquel ils sont confrontés quotidiennement. Les références en fin de chapitre sont bien choisies pour permettre l'étude de notions pertinentes, des concepts historiques classiques aux plus récents développements. On en retrouve une bonne proportion reliée aux publications de la dernière décennie, voire de l'année de publication, témoignant ainsi de la diligence du travail de révision des auteurs et de l'éditeur. L'utilisation du format pédagogique de présentation explique cependant l'absence de références reliées à de nombreuses assertions et l'utilisation de recommandations formalisées d'experts. De plus, le texte présuppose une connaissance parfois avancée de certains concepts que le lecteur devra posséder ou acquérir pour apprécier l'étendue des propos présentés. Les notions de physiologie appliquée et les données de l'examen échocardiographique en sont des exemples. On remarquera par ailleurs la description très systématisée de certaines techniques, telles que le monitorage des pressions droites par les cathéters centraux, le support ventriculaire gauche temporaire par instrumentation intra-ventriculaire ou intra-aortique, l'oxygénation par membrane extracorporelle (ECMO) veino-artérielle et les assistances ventriculaires de longue durée. 
Les algorithmes décrits dans ce manuel peuvent être légèrement différents de ceux suggérés par les sociétés canadiennes ou américaines d'anesthésiologie et de cardiologie mais les références incluses permettront au lecteur de comparer les approches. Certains médicaments en référence dans le texte ne sont pas disponibles en Amérique du Nord, ce qui ne nuit toutefois pas à la compréhension des protocoles décrits. Enfin, il aurait été intéressant, pour faciliter la lecture, de présenter un glossaire des abréviations utilisées.

En résumé, ce manuel, une première francophone, remplit bien les objectifs pédagogiques précités et sera certainement un apport apprécié des étudiants en spécialité et des cliniciens pour son survol concis des concepts essentiels de l'hémodynamique et de leurs applications.

\section{Conflit d'intérêt Aucun.}

Source de financement Aucune.

Responsabilité éditoriale Cet article a été traité par Dr Étienne de Médicis, rédacteur de la langue française, Journal canadien d'anesthésie.

Publisher's Note Springer Nature remains neutral with regard to jurisdictional claims in published maps and institutional affiliations. 